\title{
Patchy Distribution of NMDAR1 Subunit Immunoreactivity in Developing Visual Cortex
}

\author{
Christopher Trepel, ${ }^{1}$ Kevin R. Duffy, ${ }^{1}$ Victor D. Pegado, ${ }^{1}$ and Kathryn M. Murphy ${ }^{1,2}$ \\ McMaster University, Departments of ${ }^{1}$ Psychology and ${ }^{2 B}$ Biomedical Sciences, Hamilton, Ontario L8S 4K1, Canada
}

Development of ocular dominance columns is dependent on patterned retinal activity, and yet patterned activity alone cannot explain all aspects of cortical column development. Features intrinsic to the cortex have been proposed to interact with activity to guide the patterning of cortical columns (Jones et al., 1991), and the NMDA receptor, because of its role in experience-dependent plasticity, is an obvious candidate. Using immunohistochemical techniques, we found a transiently patchy distribution of the NMDA receptor 1 (NMDAR1) subunit in kitten visual cortex. Regularly spaced patches of NMDAR1immunoreactive neurons were found at the top of the cortical plate in the developing visual cortex at 2 weeks of age. At 4-5 weeks of age, the radial extent of the NMDAR1 patches spanned the supragranular layers, and by 12 weeks of age, this nonuniform pattern of NMDAR1 immunostaining was no longer apparent. Monocular visual experience prevented the expression of the NMDAR1 patches, but just $4 \mathrm{~d}$ of subsequent binocular visual experience was sufficient to promote expression of the patches. Furthermore, the NMDAR1 patches tended to be associated with the borders of ocular dominance columns. These results suggest that the degree of plasticity associated with NMDA-mediated mechanisms is elevated in local regions across the tangential extent of the visual cortex and that the NMDAR1 patches may participate in sculpting the overall arrangement of visual cortical columns.

Key words: cortical column; development; excitatory amino acid receptors; visual cortex; NMDA; ocular dominance
The columnar organization of the visual cortex emerges during development as a result of the progressive elaboration and refinement of its connections and receptive field properties. This development is dependent on visual experience early in postnatal life; disruption of vision during the critical period (Hubel and Wiesel, 1965, 1970; Olson and Freeman, 1980) can lead to a variety of activity-dependent physiological, anatomical, and behavioral changes (see Movshon and Van Sluyters, 1981). A number of neural mechanisms are involved in this plasticity (for review, see Goodman and Shatz, 1993); however, activation of the ionotropic NMDA receptor subclass of the excitatory amino acid glutamate has been implicated as a key component (Bear et al., 1990; Constantine-Paton et al., 1990; Singer and Artola, 1991; Fox and Daw, 1993; Kirkwood et al., 1995). Two properties of the NMDA receptor distinguish it from non-NMDA glutamate receptors, namely, its membrane voltage dependence and its conduction of $\mathrm{Ca}^{2+}$ ions (Goelet et al., 1986; Bliss and Collingridge, 1993). These properties are believed to make the NMDA receptor well suited for detecting coherent patterns of activity (Collingridge and Singer, 1990) of the type stimulated by normal

Received Oct. 7, 1997; revised Feb. 18, 1998; accepted Feb. 19, 1998.

This work was supported by grants from the Natural Science and Engineering Research Council (NSERC) of Canada (OGP0170583) and the Medical Research Council of Canada (MT-13624) to K.M.M. C.T. was the recipient of an NSERC postgraduate scholarship, and K.M.M. is an NSERC University Research Fellow. We thank Drs. D. G. Jones and R. J. Racine for comments on this manuscript and Bjorn Christianson for help with the Monte Carlo analysis.

C.T. and K.R.D. contributed equally to this work.

Correspondence should be addressed to Dr. Kathryn M. Murphy, Neural Organization and Plasticity Laboratory, McMaster University, Department of Psychology, 1280 Main Street West, Hamilton, Ontario L8S 4K1, Canada.

Dr. Trepel's present address: W. M. Keck Center for Integrative Neuroscience, Department of Physiology, University of California, San Francisco, 513 Parnassus Avenue, Box 0444, San Francisco, CA 94143.

Copyright (C) 1998 Society for Neuroscience $\quad 0270-6474 / 98 / 183404-12 \$ 05.00 / 0$ visual experience and ideal for participating in Hebbian synaptic modification (Hebb, 1949).

Autoradiographic studies of the distribution of the NMDA receptor in kitten visual cortex show a rise and fall in binding intensity that parallels the time course of the critical period (Bode-Greuel and Singer, 1989; Gordon et al., 1991, 1996; Reynolds and Bear, 1991). In layer IV, synaptic transmission is most sensitive to blockade of the NMDA receptor at the peak of the critical period (Fox et al., 1991). Chronic blockade of NMDA receptors in the visual cortex during the critical period disrupts development of orientation selectivity (Kleinschmidt et al., 1987), as well as changes in cortical physiology typically associated with monocular deprivation (Kleinschmidt et al., 1987; Bear et al., 1990; Daw, 1994), reverse suturing (Gu et al., 1989), and recovery of function after dark rearing (Bear et al., 1990). It has been proposed that the mechanism underlying this activity-dependent plasticity in the visual cortex is NMDA-dependent long-term synaptic potentiation (LTP) (Komatsu et al., 1981; Artola and Singer, 1987; Bear et al., 1992; Kirkwood et al., 1995, 1996). Taken together, the physiological and anatomical evidence links activation of the NMDA receptor with activity-dependent plasticity in the visual cortex.

In kitten visual cortex, various anatomical features are arranged in a patchy manner (Callaway and Katz, 1990; Schoen et al., 1990; Dyck and Cynader, 1993), and often their appearance is reminiscent of cytochrome-oxidase blobs (Horton and Hubel, 1981; Murphy et al., 1990, 1991, 1995) or ocular dominance columns (Hubel and Wiesel, 1968; LeVay et al., 1985; Anderson et al., 1988). This has led to speculation regarding the significance of these features in the development of columnar systems in the visual cortex. To date, however, there has not been a ready link between activity-dependent aspects of column development and patchy anatomical markers. With recent evidence suggesting that 
NMDA receptors may be important for emerging synapses (Aoki et al., 1994; Durand et al., 1996), it is possible that the pattern of NMDA receptors in the immature cortex interacts with correlated neural activity during the formation of ocular dominance columns in the visual cortex. To address this issue, we have mapped the laminar and tangential distribution of NMDAR1 subunit immunoreactivity in V1 during postnatal development of kittens reared with either normal visual experience or reduced binocular correlation resulting from monocular deprivation. Our main finding is that there is a transient, nonuniform distribution of NMDAR1 immunoreactivity in the supragranular layers of kitten visual cortex.

Parts of this paper have been published previously (Murphy et al., 1996; Trepel et al., 1996).

\section{MATERIALS AND METHODS}

Animals and histology. The laminar and tangential distributions of NMDAR1 subunit-immunopositive neurons were examined in the visual cortex of 13 normally reared kittens (age range, 1-12 weeks), 3 kittens monocularly deprived until 5 weeks of age, and 3 kittens monocularly deprived until 3-5 weeks of age and then allowed $4 \mathrm{~d}$ of binocular visual experience. Monocular deprivation was initiated at the time of natural eyelid opening by suturing the eyelids closed, and the fused lid margins were parted following procedures described previously (Murphy and Mitchell, 1987). Animals were killed with a lethal injection of Euthanol (sodium pentobarbital, $165 \mathrm{mg} / \mathrm{kg}$ ) and perfused transcardially with cold $0.1 \mathrm{M}$ PBS, pH $7.4\left(4^{\circ} \mathrm{C} ; 80-100 \mathrm{ml} / \mathrm{min}\right)$, until the circulating fluid was clear, followed by $2 \%$ paraformaldehyde in $0.1 \mathrm{M}$ PBS $\left(4^{\circ} \mathrm{C}\right)$ for $4 \mathrm{~min}$. The brain was removed from the cranium, and the cerebral hemispheres were resected. One hemisphere was unfolded and flattened as described previously (Olavarria and Van Sluyters, 1985; Murphy et al., 1995); the other hemisphere was blocked in the frontal plane. After being flattened and blocked, the tissue was post-fixed in cold $2 \%$ paraformaldehyde and $30 \%$ sucrose in PBS for $6 \mathrm{hr}$ and then transferred to $30 \%$ sucrose in PBS and stored overnight. The fixation protocol produced tissue that was appropriately fixed to maintain the cytostructure of the cortex while not compromising the NMDAR1 immunoreactivity of the tissue. Sections were cut on a freezing microtome at a thickness of $50 \mu \mathrm{m}$ either tangential to the pial surface from the flattened hemisphere or in the coronal plane through the intact hemisphere and were collected in PBS. Coronal sections were either reacted to visualize neurons expressing NMDAR1, stained with cresyl violet, or stained for cytochrome oxidase (CO) activity. Tangential sections were reacted for NMDAR1 immunoreactivity, except sections through layer IV from the cats that had been monocularly deprived, which were stained for CO activity (WongRiley, 1979).

Immunohistochemistry. Sections were incubated in blocking serum $[2 \%$ bovine serum albumin (BSA) and $11 \%$ normal goat serum (NGS) in PBS], then transferred to PBS, 2\% BSA, and 1\% NGS containing mouse anti-NMDAR1 (1:500) monoclonal antibody 54.1 (PharMingen, San Diego, CA), and incubated for $40 \mathrm{hr}$ at $4^{\circ} \mathrm{C}$. Immunoreactivity was visualized via the avidin-biotin process using Vectastain ABC elite kits (Vector Laboratories, Burlingame CA) and the chromogen 3,3'diaminobenzidine (DAB). Control sections were treated as described excluding the NMDAR1 antibody. Sections were then mounted onto acid-washed, gelatin-coated glass slides and coverslipped with DPX (Aldrich, Milwaukee, WI). A series of light photomicrographs (Fujichrome 64T) of the NMDAR1 immunoreactivity in areas 17 and 18 was taken using Nomarski optics and transferred to photoCD format (Eastman Kodak, Rochester, NY) or scanned directly using a high-resolution slide scanner (SprintScan 35 plus; Polaroid, Cambridge, MA). The figures were montaged after converting the images into gray levels and adjusting the contrast with the levels tool using Photoshop (Adobe Inc., San Jose, CA). Laminar boundaries for the coronal sections were determined by comparison with adjacent Nissl-stained sections (Shatz and Luskin, 1986).

Quantitative analyses. Optical staining intensity and numeric density profiles were plotted from the images of NMDAR1 immunostaining to quantify variations across laminae and within the supragranular laminae. Measurements of laminar variations in optical intensity and numeric density were made from a 500- $\mu$ m-wide sampling strip in coronal sections spanning the pial surface to the white matter. Each point on the resulting laminar optical profile was the average intensity across the $500 \mu \mathrm{m}$ strip at that laminar location. The optical intensity profiles were plotted relative to the darkest (maximum) and lightest (minimum) staining intensity along each profile. The numeric density was obtained by identifying all of the NMDAR1-immunostained neurons in the sampling strip and by calculating the number of neurons across the strip at each point along the laminar profile. Numeric densities were plotted relative to the maximum number of NMDAR1-immunostained neurons along each profile. A similar analysis was done to assess the variations in optical intensity and numeric density of NMDAR1 immunostaining within the supragranular layers. For the within-layer analyses, a tangentially oriented sampling window was used (from the top of layers II and III to the bottom of the NMDAR1 patches), and optical intensity and numeric density were quantified at each point along the tangential profile as described above. The ratio of maximum-to-minimum optical intensity and numeric density was assessed by calculating the percent contrast for each profile $[$ contrast $=($ maximum - minimum $/$ maximum + minimum $)$ $\times 100 \%$. The contrast value provides a measure of the difference between the maximum and minimum intensity or density that is not influenced by variations in overall staining or absolute numbers of neurons between sections. The spacing of the NMDAR1 patches was determined by Fourier analysis of the tangential intensity or numeric density profile. The numeric density of NMDAR1-IR neurons was also quantified in tangential sections through the superficial layers. All NMDAR1-immunopositive neurons within a sampling window from an unfolded and flattened section through the upper layers of area 17 were plotted, and a two-dimensional (2D) nearest-neighbor analysis (Voronoi polygons) was done to quantify the pattern (Guibas and Stolfi, 1985). The distribution of Voronoi polygon sizes was compared with randomly generated 2D samples to determine whether the tangential arrangement of NMDAR1 neurons was statistically different from random (Upton and Fingleton, 1985). To visualize the 2D fluctuations in density of NMDAR1-IR neurons, we calculated color-coded local density maps from the $2 \mathrm{D}$ plots of neurons in which red indicates high and blue indicates low density of NMDAR1-IR neurons. Patches were identified from the local density maps in an automated manner. The density contour that corresponded with half the maximum was plotted, and then the peak density (local maxima) within the contour was identified. Two analyses were performed of the relationship between the NMDAR1 patches and ocular dominance column borders. First, the distance from each patch to the nearest ocular dominance column border was measured, and a cumulative probability distribution was calculated. This was compared with the distributions generated with a Monte Carlo simulation to determine whether the location of the patches relative to the ocular dominance borders deviated from random. Second, the frequency of patches at different distances from the borders to the centers of the ocular dominance bands was calculated by measuring both the distance to the nearest border as well as the width of the band.

\section{RESULTS}

Light microscopy revealed NMDAR1-immunoreactive perikarya with thick and thin dendritic processes throughout the laminar extent of kitten primary visual cortex at each of the ages examined. The strongest NMDAR1 immunoreactivity was localized to cell somata and the proximal regions of apical dendrites, with the proximal portions of basilar dendrites staining less intensely and the distal dendritic regions staining lighter still. Axonal labeling could not be distinguished at any of the ages examined. The changes in the pattern of NMDAR1 immunoreactivity across laminae and within the supragranular layers in area 17 during postnatal maturation were quantified using measures of both optical staining intensity and numerical density of labeled cells. It is clear that the laminar and supragranular arrangements undergo substantial change during the course of development, both in the intensity of the neuronal staining and in the number of NMDAR1-immunoreactive neurons.

\section{Early neonatal development}

At the earliest age studied, postnatal day 7, dark bands of NMDAR1 immunostaining were apparent across the top of the developing layers II/III and across layers IV and VI (Fig. 1 $A$ ). 

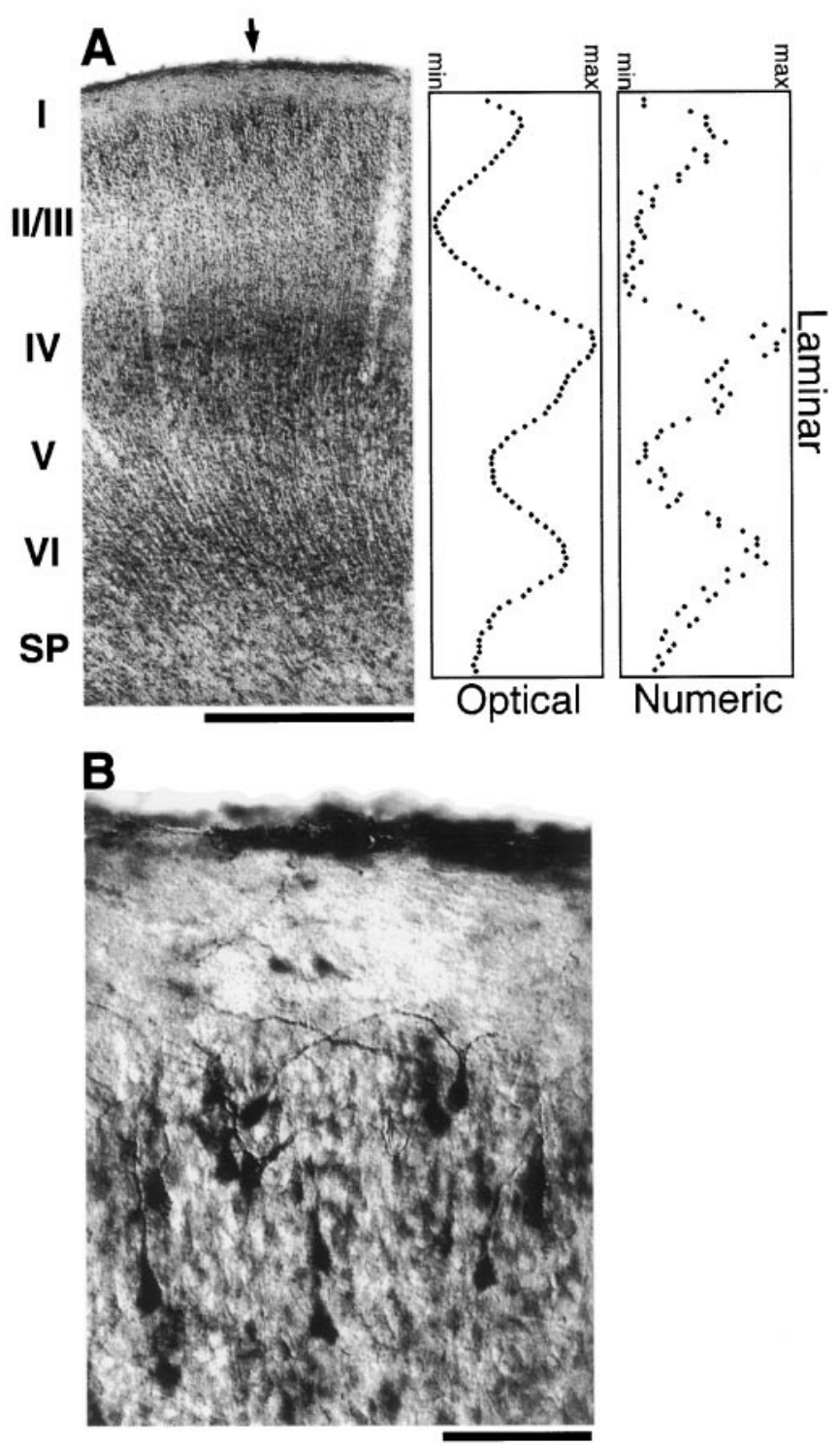

Figure 1. A, Light micrograph showing NMDAR1 immunostaining in area 17 of a 1-week-old kitten. At this age the cortex is still immature, as signified by a zone of tightly packed neurons at the top of the cortical plate. The laminar pattern of label shows dense staining in layers IV and VI as well as at the top of the cortical plate. The profiles of laminar variation in optical staining intensity and numeric density (plots on the right) had contrast values of 39 and $71 \%$, respectively. The strong correlation between these measures $(r=0.88)$ indicates that the dense label in layers IV and VI reflects both more intense staining and more labeled neurons than in the other laminae. The laminar boundaries were determined from adjacent Nissl-stained sections. $B$, Higher magnification view of layer I and the top of the cortical plate (in the region indicated by the arrow in $A$ ) showing numerous immunoreactive cell bodies and processes in the developing cortical plate, with the processes extending toward layer I. Scale bars: $A, 500 \mu \mathrm{m} ; B, 50 \mu \mathrm{m}$. $S P$, Subplate.

Perikarya of various sizes were distinctly labeled in layers II and III, with thick apical dendrites extending from pyramidal neurons toward layer I (Fig. 1B). The darkest neuropil and perikaryal staining was found in layer IV, with cells that were more punctate than were those in the upper layers. The darkest dendritic staining was localized to layers V and VI, where intensely stained somata from both laminae possessed strongly immunoreactive apical dendrites that could be traced into layer IV. In all layers, the highest levels of immunoreactivity were found in perikarya and the proximal portions of their apical dendrites.

Quantitative assessment of the laminar variations in optical staining intensity and numeric density of NMDAR1-IR neurons bore out the qualitative descriptions. The optical staining intensity profile (Fig. $1 A$ ) from layer I to the subplate showed peaks at the top of layers II and III, in layer IV, and in layer VI, with the maximum optical intensity found in layer IV. The variation from minimum to maximum optical intensity across the laminae yielded a contrast value of $39 \%$, indicating that there was a marked difference in the staining intensity between the laminae. The numeric density showed a similar laminar pattern of peaks with the largest number of NMDAR1-IR neurons in layer IV, followed by a peak in layer VI and at the top of layers II and III (Fig. 1A). The ratio between the minimum and maximum numeric density of NMDAR1-IR neurons across layers was very pronounced, reflected in a numeric contrast of $71 \%$. The laminar variations in optical staining intensity and numeric density were strongly correlated $(r=0.88)$, indicating that at 1 week of age the dark bands of NMDAR1 immunoreactivity observed in layers II/III, IV, and VI were the product of both larger numbers of NMDAR1-IR neurons and intense staining.

As early as 1 week of age, some aggregates of darkly stained NMDAR1-immunopositive pyramidal cells were observed in the superficial aspect of the developing cortical plate (Fig. 1A, arrow). Both their distinctiveness as well as their laminar position suggest that they may be early signs of the NMDAR1 patches evident in older kittens.

At 2 weeks of age, the laminar variation in NMDAR1 immunoreactivity in area 17 was very similar to the pattern observed at 1 week of age. Dense NMDAR1 immunoreactivity was observed at the top of layers II and III, in layer IV, and in layer VI. A distinct difference was apparent, however, in the labeling pattern within the superficial tier of layers II and III (Fig. 2A, asterisk), where dense patches of NMDAR1 immunostaining were found in area 17. Examination of the staining pattern in layer II revealed numerous NMDAR1-IR pyramidal cells and their processes, as well as slightly darker neuropil staining, within a patch (Fig. 2B, filled arrowhead) relative to an interpatch region (Fig. 2B, open arrowhead). A gradient of labeling intensity was also apparent, with pyramidal cells within a patch more intensely labeled than neighboring cells either within layer II or proximally located in layers I or III. This type of patchiness in the expression of NMDAR1 was not observed in either the granular or the infragranular layers.

Analysis of the tangential variation in NMDAR1 immunoreactivity within layer II at 2 weeks of age revealed regular fluctuations in both the optical staining intensity and the numeric density of NMDAR1-IR neurons. A clear waxing and waning of the tangential optical staining intensity was found (Fig. 2A). Quantification of the fluctuations in intensity yielded an optical contrast of $38 \%$ between the maximum and minimum staining intensities. Moreover, analysis of the local variations in the density of NMDAR1-IR neurons within layer II demonstrated regular fluctuations in the number of immunopositive neurons (Fig. $2 A$ ), with a numeric contrast of $55 \%$ between the patch and interpatch regions. The large contrast value supports the observation that there are greater numbers of NMDAR1immunoreactive neurons within the patches relative to the interpatch regions (Fig. 2B). The tangential profiles of numeric density and optical intensity were strongly correlated $(r=0.86)$, and Fourier analysis of the profiles indicated an interpatch spac- 

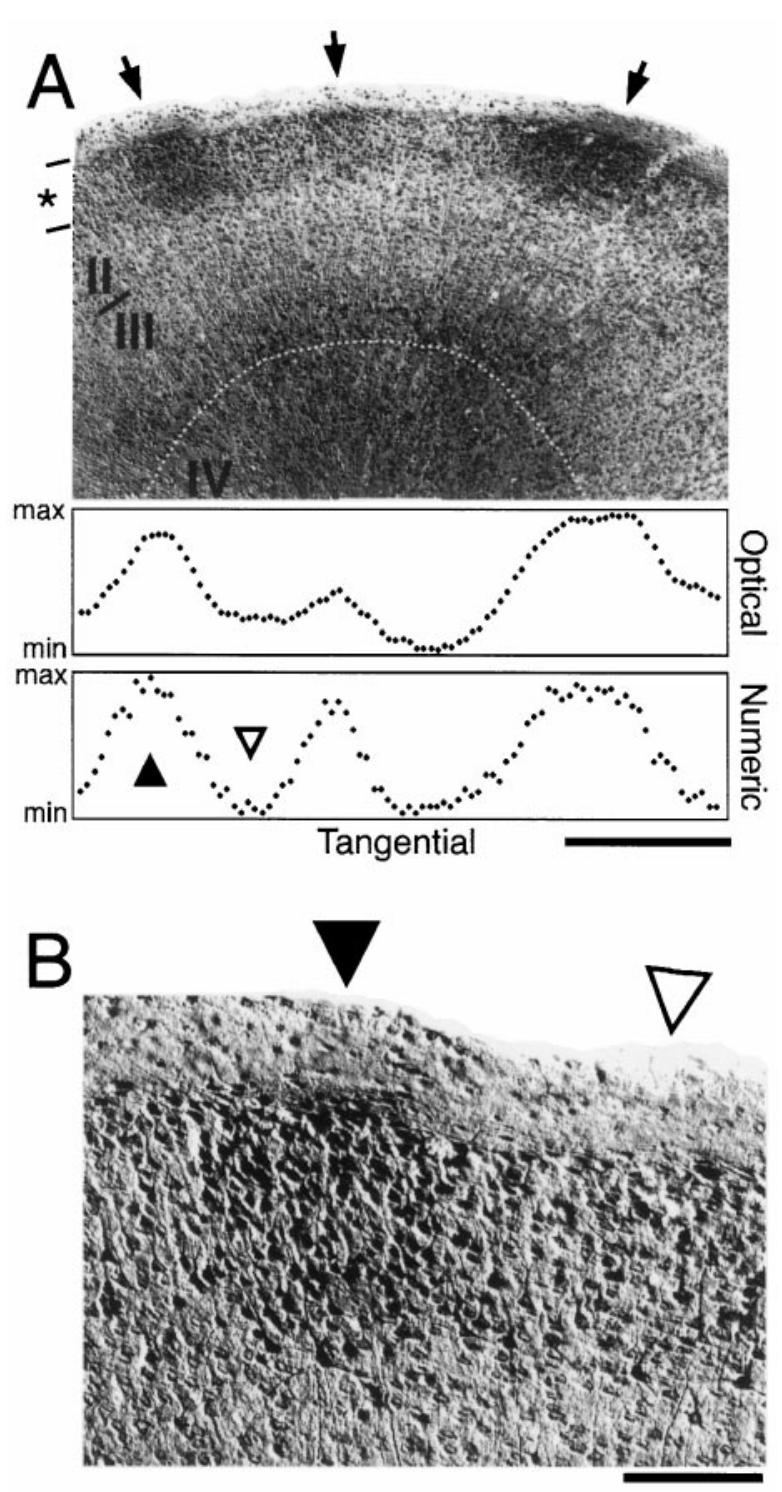

Figure 2. NMDAR1 patches are clearly visible at 2 weeks of age. $A$, At low magnification, these patches (arrows) may be seen in layer II, with layer IV continuing to show heavy, uniform immunostaining. Tangential optical intensity and numeric density profiles (plots on the bottom) through these patches show a pattern of large, regular fluctuations, and these two measures are strongly correlated $(r=0.86)$. $B$, A high magnification micrograph of the left-most patch seen in $A$ is shown. It is apparent that immunoreactive layer II pyramidal cells comprise these patches. Note the well-defined apical and basal dendritic processes. Scale bars: $A, 500 \mu \mathrm{m} ; B, 100 \mu \mathrm{m}$.

ing of $677 \mu \mathrm{m}$. These results demonstrate that the NMDAR1 patches in layer II are comprised of both more intense staining and more NMDAR1-IR neurons relative to the interpatch regions.

\section{Peak of the critical period}

Several changes in the laminar pattern of NMDAR1 immunostaining were apparent at 4 weeks of age (Fig. $3 A$ ). Layer I of 4-week-old kittens continued to show sparse NMDAR1 immunoreactivity punctuated by immunoreactive cells. Neurons in layers II and III at 4 weeks of age were well defined, and considerable variability in size notwithstanding, the majority were pyramidal in shape. Almost all had well-stained, thick apical processes, with
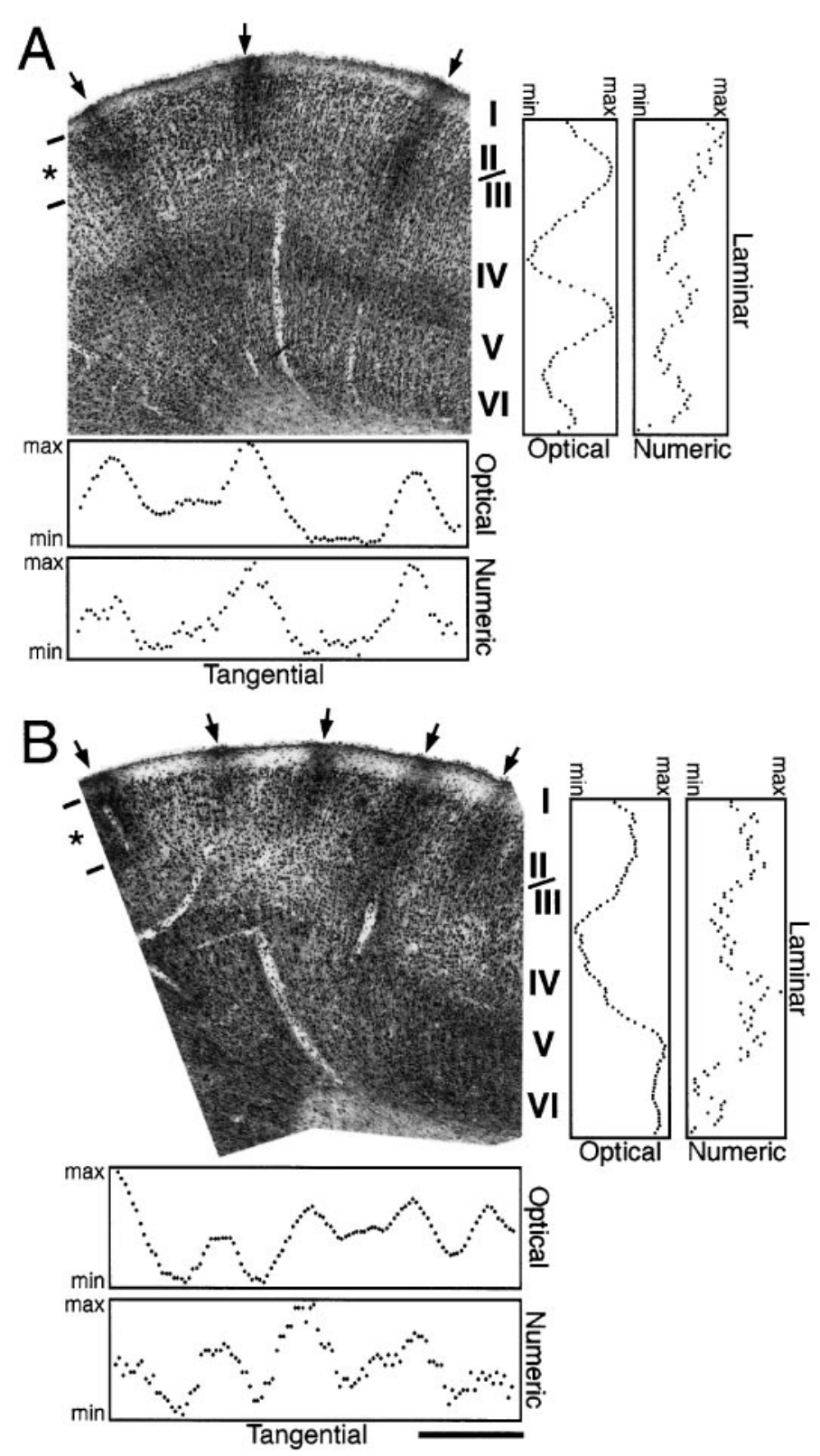

Figure 3. NMDAR1 immunoreactivity in area 17 at 4 weeks $(A)$ and 5 weeks $(B)$ of age with profiles of both the laminar (right) and the supragranular tangential (bottom) optical staining intensities and numeric densities of NMDAR1 immunoreactivity. $A$, By 4 weeks of age, the radial extent of the densely labeled NMDAR1 patches (arrows) has lengthened to encompass layers I-III and encroaches into the most superficial aspect of layer IV. The lower half of layer IV also demonstrates dense immunolabeling. These laminar and tangential patterns are reflected in the profiles. $B$, Light micrograph of the pattern of NMDAR1 immunostaining at 5 weeks of age is shown. Prominent, densely labeled NMDAR1 patches (arrows) can be seen to extend through the supragranular layers. Although the correlation between the tangential optical intensity and numeric density through the patches in layers II and III was strong $(r=0.81)$, the laminar profiles were less well correlated $(r=0.49)$, indicating that neuropil staining was contributing to the laminar staining pattern. Scale bar, $500 \mu \mathrm{m}$.

greater numbers than at early ages also showing clear, moderately labeled thick and thin basilar processes extending horizontally. Several radially oriented bundles of immunoreactive processes were apparent in upper layer II and extending into layer I, 
whereas the apical dendrites of many lower layer II and upperto-mid layer III cells could be followed into the superficial extent of layer II. Fewer labeled cells in layer IV had readily distinguishable processes relative to cells in layers II and III. Concurrently, the dark band of immunostaining observed previously throughout layer IV was concentrated in lower layer IV, resulting in the upper region of the granular layer (IVA) appearing light by comparison. Large, well-labeled pyramidal cells with thick apical and basilar dendrites were found in layer V. The apical dendrites of the large layer $\mathrm{V}$ pyramidal cells could be traced through layer IV with some extending into layer III. Layer VI cells generally appeared as smaller pyramids with thinner and more lightly stained processes than those seen in layers III or V. The neuropil of layer VIB was more intensely immunostained, and its pyramidal cells appeared slightly darker compared with layer VIA cells.

At 4 weeks of age, analysis of the laminar variation in optical staining intensity showed peaks in staining intensity in layers II and III, lower layer IV, and lower layer VI (Fig. 3A). The optical contrast of these laminar variations was $13 \%$, indicating that the laminar differences in staining intensity were less pronounced than were those found in younger kittens. The profile of laminar variations in numeric density also showed peaks in layers II and III, layer IV, and layer VI; however, the correlation between the optical intensity and numeric density profiles was less than that found in younger kittens $(r=0.49)$. The reduced correlation indicates that the laminar variations in staining intensity do not simply reflect differences in the number of NMDAR1-IR neurons but instead must also reflect differences in the level of neuropil staining.

The NMDAR1 patches appeared as radially oriented columns at 4 weeks of age extending from layer I through layers II and III, with some dipping into upper layer IV (Fig. $3 A$ ). These patches were clearly reflected in the pattern of strong peaks and troughs in the tangential optical intensity and numeric density profiles (Fig. 3A, asterisk) through layers II and III (optical contrast, 25\%; numeric contrast, $43 \%$ ). The peak-to-peak spacing of the numeric profile in Figure $3 A$ was $798 \mu \mathrm{m}$. The optical intensity and numeric density were strongly correlated $(r=0.81)$, indicating that patches of high NMDAR1 immunostaining also contained more NMDAR1-IR neurons than were found in the less intensely stained interpatch regions.

The laminar pattern of NMDAR1-immunopositive cell types in area 17 at 5 weeks of age (Fig. $3 B$ ) was very similar to that of the cell types labeled at 4 weeks of age. Quantification of this laminar pattern showed progressive development toward the mature arrangement. The highest level of staining, as quantified by optical staining intensity, was found in the infragranular layers (Fig. $3 B$ ), whereas the lowest overall staining level was in layer IV. The numeric density profile showed the peak density of NMDAR1-IR neurons to be in lower layer IV and layer V, followed by the supragranular layers, whereas the minimum numeric density was in layer VI. There was a clear divergence in layer VI between the optical intensity and numeric density, and this difference was reflected in the very low correlation between the laminar profiles $(r=0.10)$. Closer examination of the labeling in layer VI revealed that the increased optical intensity was a result of an increase in neuropil staining. This divergence between optical intensity and numeric density profiles speaks directly against the notion that there is a simple relationship between these two measures or that one always predicts the other and underscores the importance of the numeric analysis in combination with optical density measures.
The supragranular NMDAR1 patches in area 17 at 5 weeks of age extended from layer I through to layer III, with some clearly encroaching into the superficial aspect of layer IV (Fig. $3 B$ ). There was no patchiness observable in the infragranular layers. Closer examination of the patches at 5 weeks of age using Nomarski optics revealed an increased density of fine NMDAR1 immunostained processes arising from layer II/III pyramidal cells and extending into layer I relative to an interpatch region. These processes, as well as darker neuropil staining, contributed to the darker immunostaining in layer I within a NMDAR1 patch. Analysis of the NMDAR1 patches in supragranular layers of area 17 revealed periodic fluctuations in both optical staining intensity (optical contrast, 23\%) and numeric density (numeric contrast, $38 \%$ ) of NMDAR1-IR neurons (Fig. 3B). Furthermore, the tangential profiles of numeric density and optical intensity within layers II and III were correlated $(r=0.57)$. Fourier analysis of the numeric density profile had one major peak corresponding to a spacing of $535 \mu \mathrm{m}$ between the NMDAR1 patches in this example.

Analysis of the local density of NMDAR1 neurons from unfolded and flattened sections through layers II and III of area 17 showed the 2D pattern of fluctuations in numeric density at 4 weeks (Fig. 4A,C) and 5 weeks (Fig. $4 B, D$ ) of age. Two sets of analyses were performed on the 2D plots of NMDAR1-IR neurons. First, a nearest-neighbor analysis was performed (Voronoi polygon) to characterize the distribution of polygon areas for the NMDAR1 plots (Fig. $4 A, B$ ) and to compare these with randomly generated plots. All of the distributions of NMDAR1-defined polygon areas were different from random $(p<0.001)$. Second, color-coded 2D plots of local numeric density were calculated (Fig. $4 C, D$ ) and showed a regular arrangement of patches of high density (red regions) interspersed with regions of lower density. The pattern of high density NMDAR1 patches is reminiscent of the 2D pattern of cytochrome oxidase blobs in cat visual cortex.

NMDAR1 immunostaining of area 18 at 5 weeks of age showed the same general features described above for area 17. Pronounced patches of NMDAR1 immunoreactivity were apparent in the supragranular layers in area 18 at $4-5$ weeks of age (Fig. 5 , asterisk). The patches in area 18 extended from layer I through layer III, and as in area 17, densely labeled pyramidal somata, dendritic processes, and neuropil distinguished the NMDAR1 patches from the intervening regions. The profiles of optical intensity and numeric density within the supragranular layers showed periodic fluctuations (Fig. 5) that had maximum to minimum differences equivalent to 30 and $33 \%$, respectively. These profiles were closely correlated $(r=0.89)$, and Fourier analysis of this numeric density profile indicated that the spacing between NMDAR1 patches was $568 \mu \mathrm{m}$. The relationship between staining intensity and number of NMDAR1-IR neurons indicated that the patches in area 18 were comprised of more darkly labeled neurons relative to the interpatch regions, similar to the relationship found in area 17, and that this nonuniform distribution of the NMDAR1 subunit is a robust feature of both the primary and secondary visual cortices at these ages.

\section{Late critical period to maturation}

The overall intensity of NMDAR1 immunoreactivity in areas 17 and 18 at 8 weeks of age was similar to that seen at 5 weeks of age (Fig. 6A). The composition of NMDAR1-IR neurons in the supragranular layers had not changed significantly from 5 weeks of age. Layer IV showed the lightest staining, and the perikarya of this layer were more lightly stained and sparser than at 4 or 5 

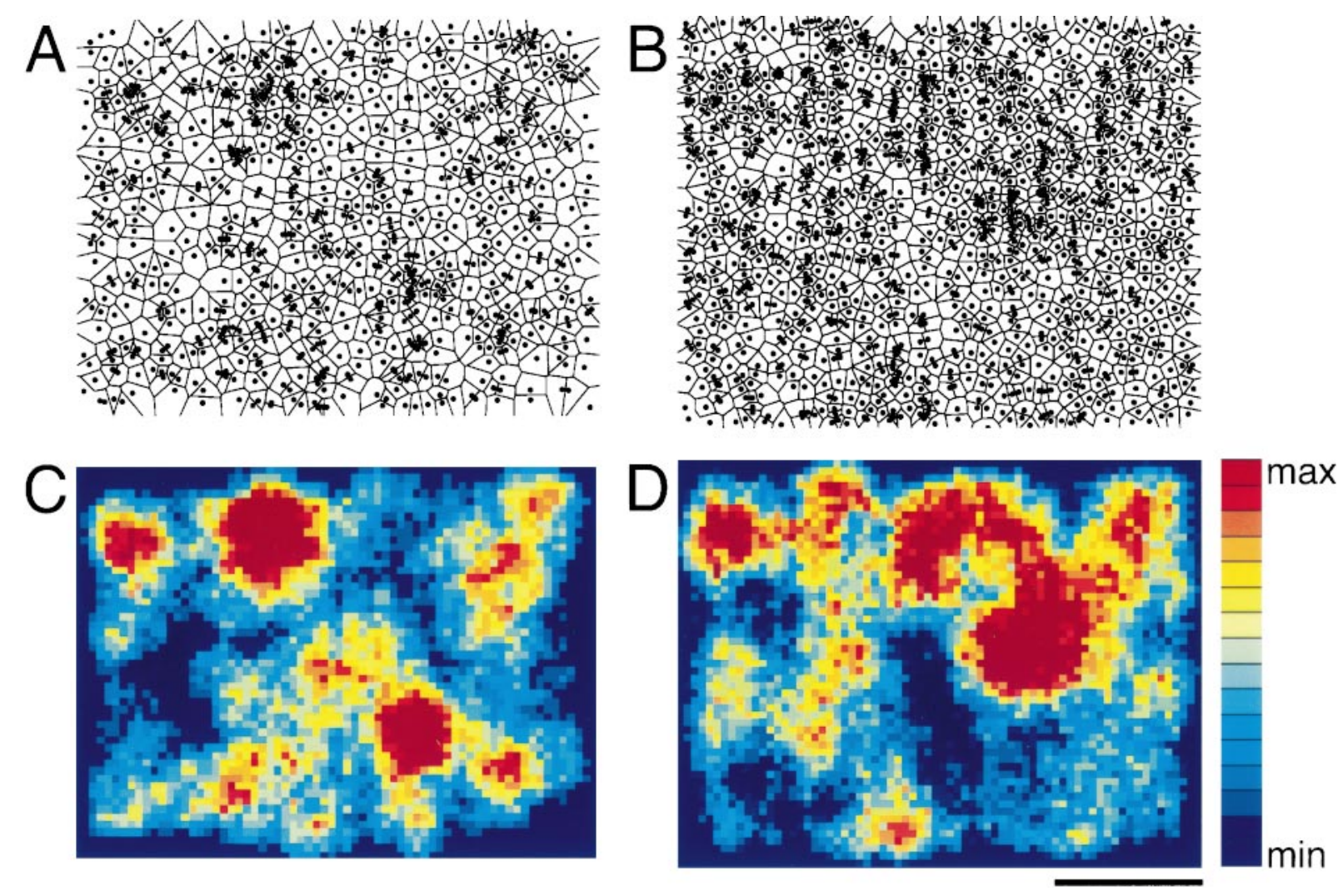

Figure 4. Nearest-neighbor analyses of the 2D arrangement of NMDAR1-immunostained neurons from unfolded and flattened sections through the supragranular layers of area 17. $A, B$, All of the NMDAR1-IR neurons in the sampling window were located (dots), and the Voronoi polygon around each neuron, in which every point inside the polygon is closer to the neuron it encircles than to any other neuron in the plot, was calculated $(A, 4$ weeks of age; $B, 5$ weeks of age). The distributions of polygon areas were significantly different from the distributions for randomly generated points $(p<0.001)$. $C, D$, Color-coded local density maps from the above plots of NMDAR1-IR neurons are shown $(C$ is from $A ; D$ is from $B)$, in which red indicates a higher and blue indicates a lower density of NMDAR1-IR neurons. There is a regular 2D arrangement of high density NMDAR1 patches. Scale bar, $500 \mu \mathrm{m}$.

weeks of age. The pattern of NMDAR1 immunoreactivity in the infragranular layers showed darker bands through layers $\mathrm{V}$ and VIB. Layer V and VIB had many well-defined NMDAR1-IR pyramidal cells with well-labeled apical processes that extended toward the pial surface.

Some NMDAR1 patches were present in the supragranular layers of area 17 at 8 weeks of age; however, they were located only around the crown of the postlateral gyrus and up to the border with area 18 (Fig. 6A). As observed in sections from younger kittens, the patches were composed of densely labeled layer II/III pyramidal cells encapsulated by slightly darker neuropil staining. Farther away from the border between areas 17 and 18, down the medial bank of area 17 and within area 18, NMDAR1 immunostaining in the upper layers was dark and did not exhibit obvious patchiness.

By 12 weeks of age, the features of the NMDAR1 immunostaining in area 17 of the cat had undergone a number of changes relative to earlier time points. Layer IV neurons were very lightly labeled, whereas neurons in the supragranular and infragranular layers continued to be well labeled. In particular, dark NMDAR1 immunostaining was observed in layer VIB in area 17. There was very little neuropil immunoreactivity in layer I at this age, except for a small number of sparsely distributed layer II apical dendrites that extended into layer I.

The most striking difference observed at 12 weeks of age was the complete absence of the nonuniform, patchy NMDAR1 immunostaining characteristic of the supragranular layers at earlier

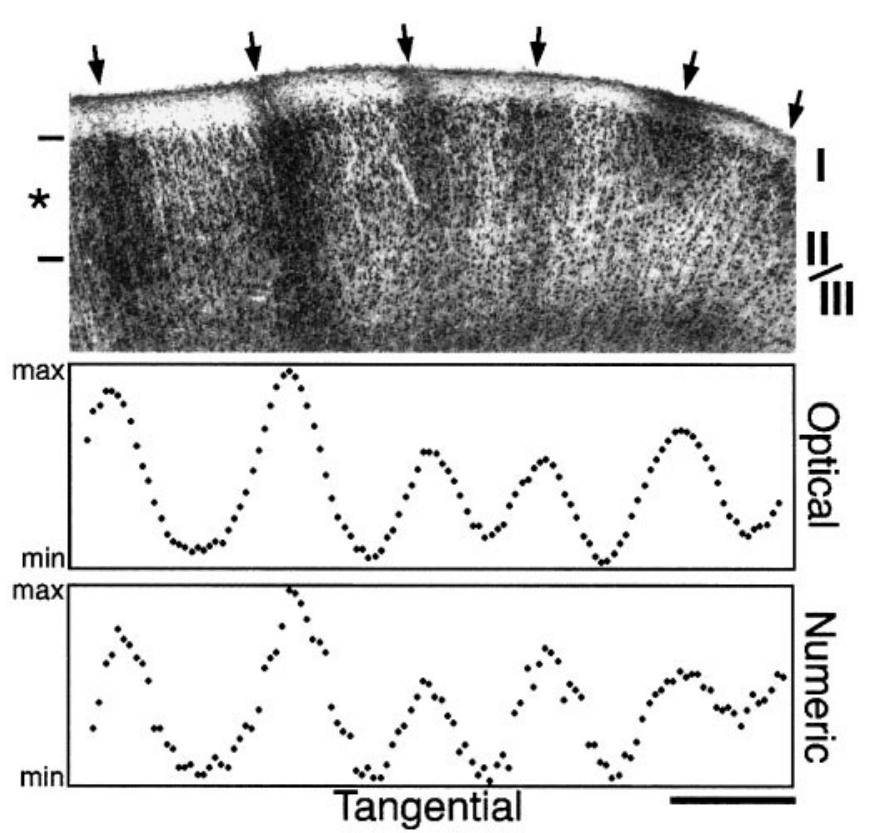

Figure 5. In the superficial layers of area 18, densely labeled patches of NMDAR1-immunostained neurons (arrows) were apparent at 5 weeks of age, extending from layer I to the top of layer IV. The tangential profiles of optical intensity and numeric density (bottom) through the NMDAR1 patches showed regular fluctuations. Scale bar, $500 \mu \mathrm{m}$. 


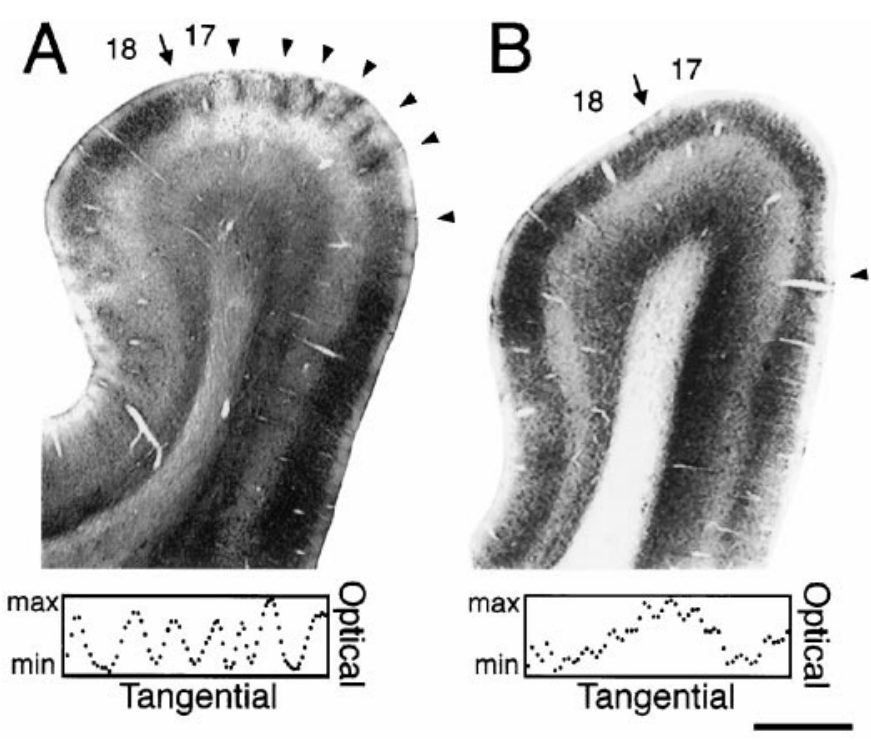

Figure 6. A, At 8 weeks of age, the optical intensity analysis [from the border (arrow) between areas 17 and 18 to the right-most arrowhead in area 17] revealed that the supragranular layers of area 17 continued to have NMDAR1 patches (arrowheads), mainly near the crown of the area 17 gyrus, whereas examination of the medial bank revealed dense, uniform immunostaining. The NMDAR1 labeling in area 18 exhibited dense, uniform labeling in layers II and III. $B$, At 12 weeks of age, the optical intensity analysis showed that NMDAR1 patches were no longer apparent in the supragranular layers of areas 17 or 18 . Scale bar, $500 \mu \mathrm{m}$.

ages. The nonpatchy distribution observed along the medial bank of area 17 at 8 weeks of age had expanded to cover the entirety of area 17 (Fig. 6B). Analysis of optical intensity through the supragranular layers did not show the large, periodic fluctuations in intensity observed in younger kittens, thereby reaffirming the qualitative observations that the mature pattern of NMDAR1 immunostaining was not patchy.

\section{Monocular deprivation and subsequent binocular vision}

The pattern of NMDAR1 immunostaining in area 17 after monocular deprivation to 5 weeks of age was different from that observed in kittens reared with normal visual experience. Most notably, no patchiness was observed in any layer of the visual cortex (Fig. 7A) in any of the monocularly deprived kittens. The overall intensity of the labeling was reduced, and the staining of individual neurons was less distinct when compared with that of normally reared kittens. In the supragranular layers, there were numerous lightly labeled perikarya, most of which were pyramidal in shape. Few of these neurons had stained dendritic processes, and when stained apical dendrites were observed, the NMDAR1 immunostaining was apparent only on the most proximal portion of the dendrite. The small, granular-shaped neurons in the upper portion of layer IV were very lightly labeled, whereas a darker band of immunostaining was observed across the ventral aspect of layer IV and through layer V. This band represented immunostaining of the perikarya and the most proximal portions of the apical dendrites and was the darkest NMDAR1 immunostaining observed in area 17 after 5 weeks of monocular deprivation. Small, lightly stained, pyramidal-shaped perikarya characterized the NMDAR1 immunostaining in layer VI, with few labeled processes present. Quantitative analysis of the distribution of NMDAR1 immunostaining in layers II and III (Fig. 7A) showed no periodic variation of the optical intensity or the numeric

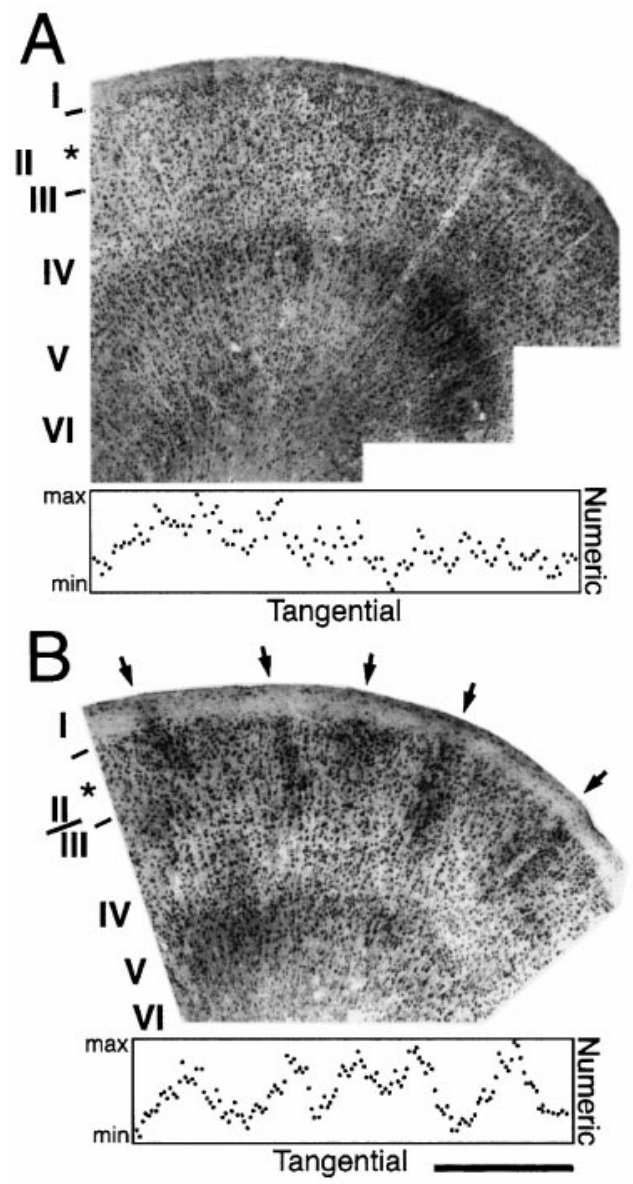

Figure 7. $A$, The pattern of NMDAR1-IR in the visual cortex after monocular deprivation from eye opening to 5 weeks of age. The labeling was lighter than that observed in normally reared animals and did not exhibit patchiness in any layers. Quantitative analysis of the numeric density of NMDAR1-immunostained neurons (bottom) through the supragranular layers (asterisk) showed no regular fluctuations in density. $B$, The pattern of NMDAR1-IR in the visual cortex when $4 \mathrm{~d}$ of binocular vision was introduced after monocular deprivation to 5 weeks of age. There were obvious patches (arrows) of NMDAR1 immunostaining in the supragranular layers, and quantitative analysis of the numeric density of NMDAR1-IR neurons (bottom) across layer II/III (asterisk) showed regular periodic fluctuation with a spacing of $491 \mu \mathrm{m}$ between the NMDAR1 patches in this example. Scale bar, $500 \mu \mathrm{m}$.

density in the visual cortex of any monocularly deprived kittens. Thus, monocular deprivation from the time of eye opening reduced the overall intensity of NMDAR1-IR in the visual cortex and changed the pattern of NMDAR1-immunostained neurons in the supragranular layers.

The introduction of $4 \mathrm{~d}$ of binocular vision after monocular deprivation resulted in dark, periodic patches of NMDAR1immunostained neurons in the supragranular layers (Fig. $7 B$ ) of all the kittens reared in this manner. NMDAR1-IR neurons in the supragranular layers were well defined, with many more darkly labeled neurons and processes than were observed immediately after termination of monocular deprivation. Interestingly, the patches observed after $4 \mathrm{~d}$ of binocular vision were in the same region of area 17 as those observed at 8 weeks of age (Fig. $6 A$ ), namely, around the crown of the postlateral gyrus up to the border with area 18. Analysis of these patches showed regular periodic fluctuations in both the optical intensity (contrast, 35\%) and numeric density (contrast, 39\%) of NMDAR1-IR neurons 


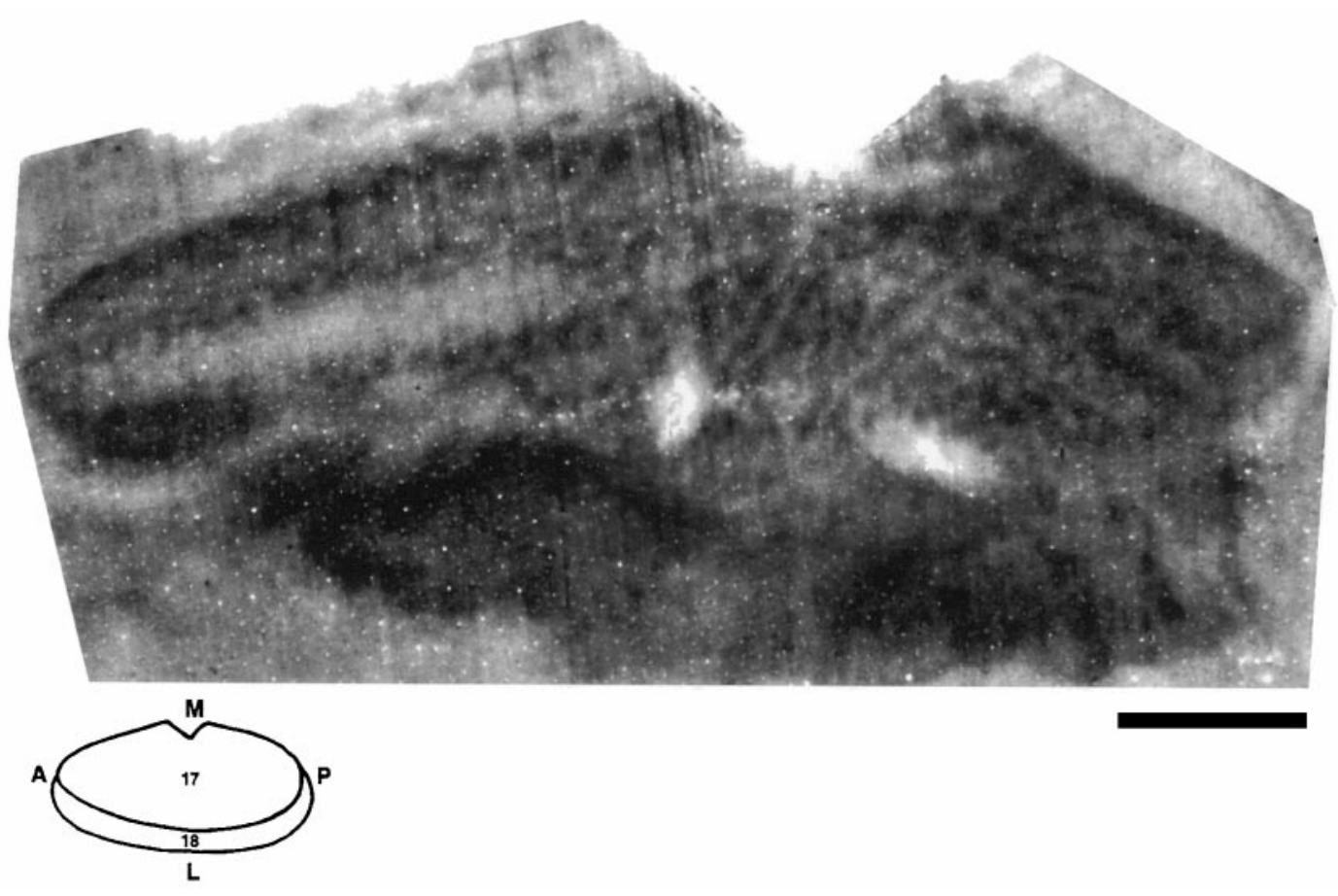

Figure 8. The 2D pattern of ocular dominance columns in layer IV after monocular deprivation to 5 weeks of age and $4 \mathrm{~d}$ of binocular vision. The sections were stained for $\mathrm{CO}$ activity, and the darker bands represent the columns of the initially nondeprived eye. The $2 \mathrm{D}$ arrangement of ocular dominance columns is already adult-like at this age. Inset shows the relationship between areas 17 and 18 in flattened sections. Scale bar, $5 \mathrm{~mm}$. $A$, Anterior; $M$, medial; $P$, posterior; $L$, lateral. with an average spacing of $486 \mu \mathrm{m}$ between the patches. This spacing was similar to that observed in normally reared kittens and approximately the width of one eye's ocular dominance columns.

Finally, the arrangement of NMDAR1 patches was compared with the underlying pattern of ocular dominance columns. After monocular deprivation and $4 \mathrm{~d}$ of binocular vision, the LGN laminae receiving input from the initially nondeprived eye were more darkly stained for CO activity, and there was a clear pattern of light and dark ocular dominance bands (Murphy et al., 1995) in layer IV of CO-stained sections (Fig. 8). Using radial blood vessels, we aligned the 2D map of NMDAR1-IR neurons with the pattern of ocular dominance columns. Visual comparison of the map of NMDAR1 patches (Fig. $9 A$ ) with the ocular dominance columns (Fig. 9B) showed that some NMDAR1 patches (red regions) were near the borders of ocular dominance columns; however, others were not (Fig. 9C). To test whether these features were related, we performed two quantitative analyses. First, the NMDAR1 patches were identified in an automated manner (see Materials and Methods), and the distance to the nearest ocular dominance border (Fig. 9D) was measured and compared with Monte Carlo simulations. This showed that the NMDAR1 patches and ocular dominance columns were not independent ( $p<0.005$; Wilcoxon test). Second, the number of NMDAR1 patches in different regions of the ocular dominance columns, from the border to the center, was counted. The frequency distribution showed a higher incidence of NMDAR1 patches near the borders $(\sim 32 \%)$ and fewer near the centers $(\sim 9 \%)$ of ocular dominance columns ( $p<0.00001 ; \chi^{2}$ test) (Fig. 10). These analyses indicate a tendency for NMDAR1 patches to lie preferentially near ocular dominance borders and to avoid the centers.

\section{DISCUSSION}

In this study, we have used a monoclonal antibody to the NMDAR1 subunit to determine the laminar and tangential distribution of this key component of experience-dependent plastic- ity during postnatal development of kitten visual cortex. We found a new feature of the tangential organization of the supragranular layers. Dense, regularly spaced patches of NMDAR1immunoreactive neurons were observed in the supragranular layers of V1 and V2 of kittens reared with binocular visual experience. The NMDAR1 patches were clearly visible at the top of the developing cortical plate at 2 weeks of age; by 4 weeks of age, their radial extent had lengthened to encompass layers I-III; and by 12 weeks of age, they were no longer apparent. Although the most ventral aspect of the NMDAR1 patches may dip into the superficial tier of layer IV, there was no obvious patchiness in layer IV at the ages studied. Furthermore, the patches were not expressed after early monocular deprivation. This pattern of NMDAR1 immunostaining extends an observation by Aoki et al. (1994) who reported clusters of NMDAR1-immunostained apical dendrite bundles below the marginal zone in developing rat visual cortex [see Aoki et al. (1994), their Fig. 6C].

There are several methodological factors that contributed to the identification of NMDAR1 patches in this study. In contrast to the much thinner sections that must be used in autoradiographic preparations (e.g., Bode-Greuel and Singer, 1989; Smith and Thompson, 1994), the use of 50- $\mu$ m-thick sections in this study permitted a greater proportion of the cell somata to remain intact and labeled. Moreover, autoradiographic labels possessing only moderate binding affinities, such as $\left[{ }^{3} \mathrm{H}\right]$ glutamate or [ ${ }^{3}$ H]MK-801 (e.g., Reynolds and Bear, 1991; Rosier et al., 1993; Gordon et al., 1996), cannot achieve the same level of precision afforded by the high-affinity immunocytochemical labels used in the present study (Huntley et al., 1994; Siegel et al., 1994). Finally, the use of a monoclonal antibody provides the cellular resolution necessary for visualizing NMDAR1-labeled neurons and for quantifying their distribution.

The laminar pattern of development of NMDAR1 immunostaining is in accordance with both homogenate and in situ autoradiographic studies of NMDA receptor development 
A

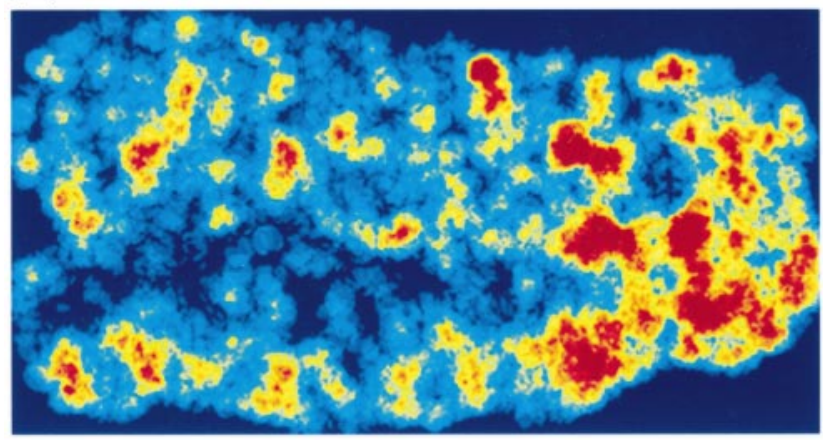

C

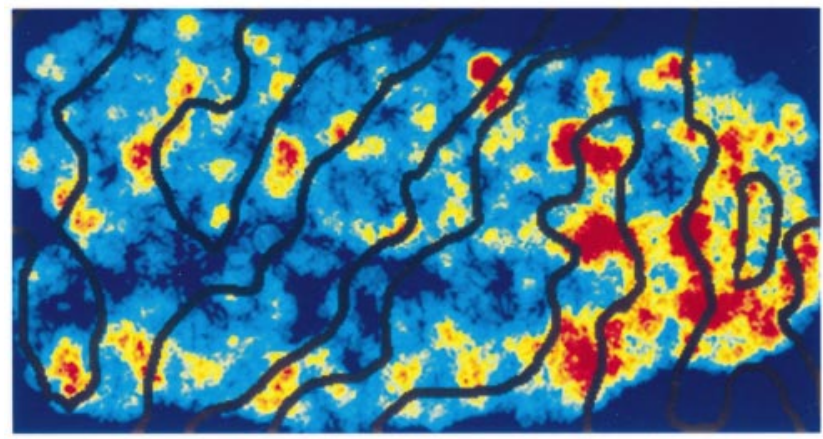

B

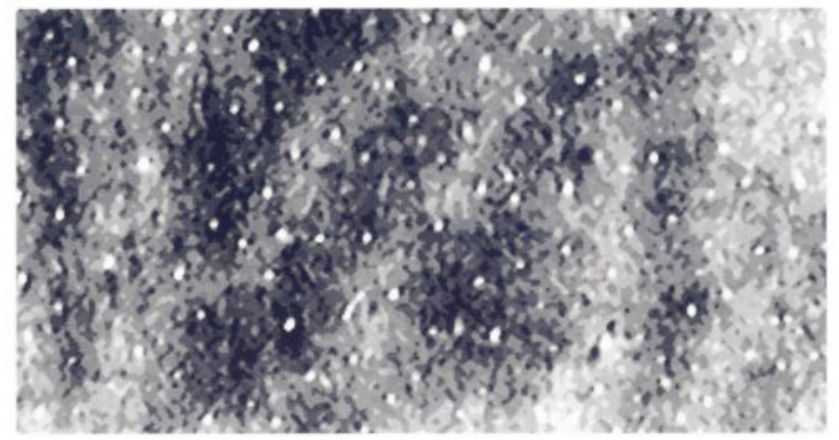

D

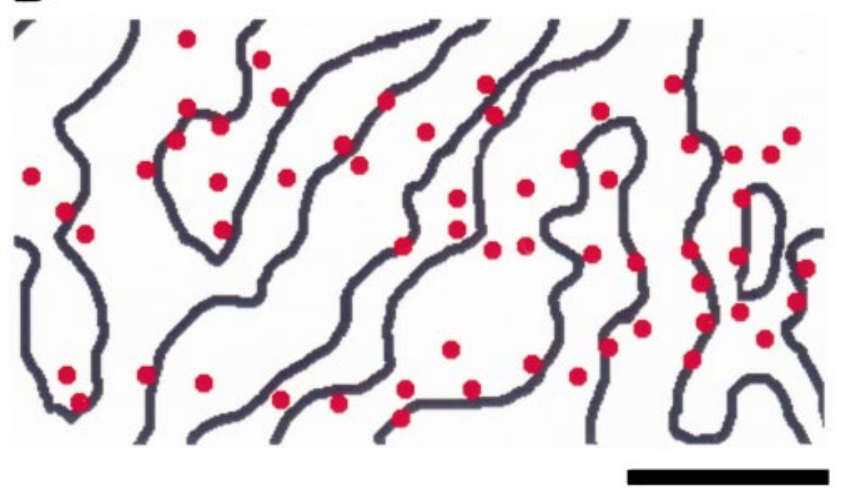

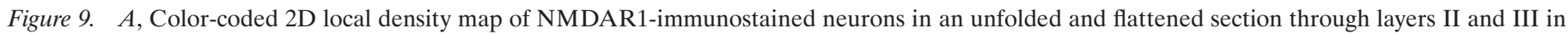

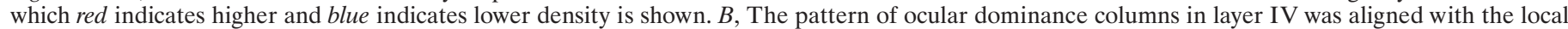

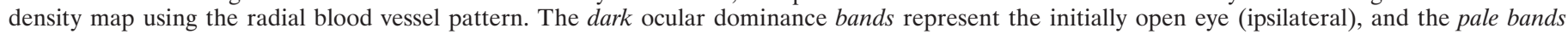

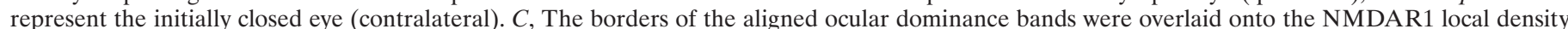

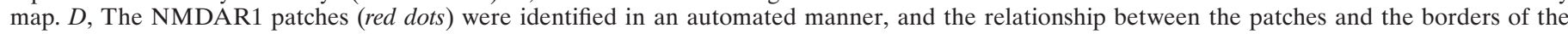

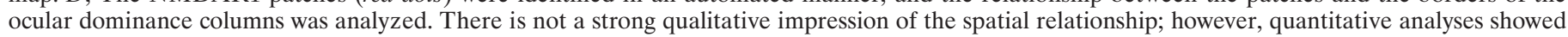
that the NMDAR1 patches tend to avoid the centers of ocular dominance columns (see Fig. 10).

(Greenamyre et al., 1985; Bode-Greuel and Singer, 1989; Rosier et al., 1993; Gordon et al., 1996). Initially, the highest levels of NMDAR1 subunit labeling are in layer IV and then subsequently within the superficial layers of the visual cortex. These laminar results agree with previous immunohistochemical studies examining the development of the NMDA receptor in rat, cat, and ferret visual cortex (Aoki et al., 1994; Aoki, 1997; Catalano et al., 1997). In addition, it is clear that during the early stage of development, the laminar pattern of NMDAR1 immunostaining primarily reflects the number of labeled neurons and the intensity with which they are stained. In the adult, however, neuropil staining contributes to the laminar appearance of NMDAR1 immunoreactivity. This was especially true for layer VIB that appeared as a darker band in the adult because of the more intense neuropil staining in that layer.

\section{Relationship to visual cortical LTP}

The abrupt increase at 2 weeks of age in the density of supragranular NMDAR1-IR, both inside and outside the patches, is consistent with the synaptic electrophysiology of this system. NMDA-dependent LTP has been shown to be induced more easily both in supragranular visual cortical neurons (Komatsu et al., 1988; Kirkwood and Bear, 1994) and in immature neocortex (Artola and Singer, 1987; Perkins and Teyler, 1988; Komatsu and Toyama, 1989). Several studies (Tsumoto and Suda, 1979; Komatsu et al., 1981, 1988), however, have demonstrated that slices of kitten visual cortex younger than 2 weeks of age show resistance to the induction of supragranular LTP. Furthermore, LTP induction in the supragranular layers has been reported to occur with diverse probabilities (e.g., Komatsu et al., 1988; Kato et al., 1991; for review, see Teyler et al., 1990), and it is possible that the patchy expression of NMDAR1-IR reported here may contribute to this variability. The dependence of neocortical LTP induction on the activation of NMDA receptors (Sutor and Hablitz, 1989; Artola and Singer, 1990; but see Komatsu et al., 1991) suggests that the probability of LTP induction across the extent of the visual cortex could be affected by the position of the electrode relative to an NMDAR1 dense patch.

\section{Relationship to visual cortical columns}

Activation of the NMDA receptor is a necessary component of the experience-dependent development of ocular dominance columns (Kleinschmidt et al., 1987), and NMDA receptor channel function decreases during the critical period as the capacity for columnar rearrangement diminishes (Fox et al., 1991). Based on these findings and the present results, an obvious question is whether the patches are related to ocular dominance columns. Appearance of the NMDAR1 patches was disrupted by early monocular deprivation, suggesting that expression of NMDAR1 patches may depend on binocular visual experience that leads to normal segregation of ocular dominance columns. We tested this possibility by introducing a short period 


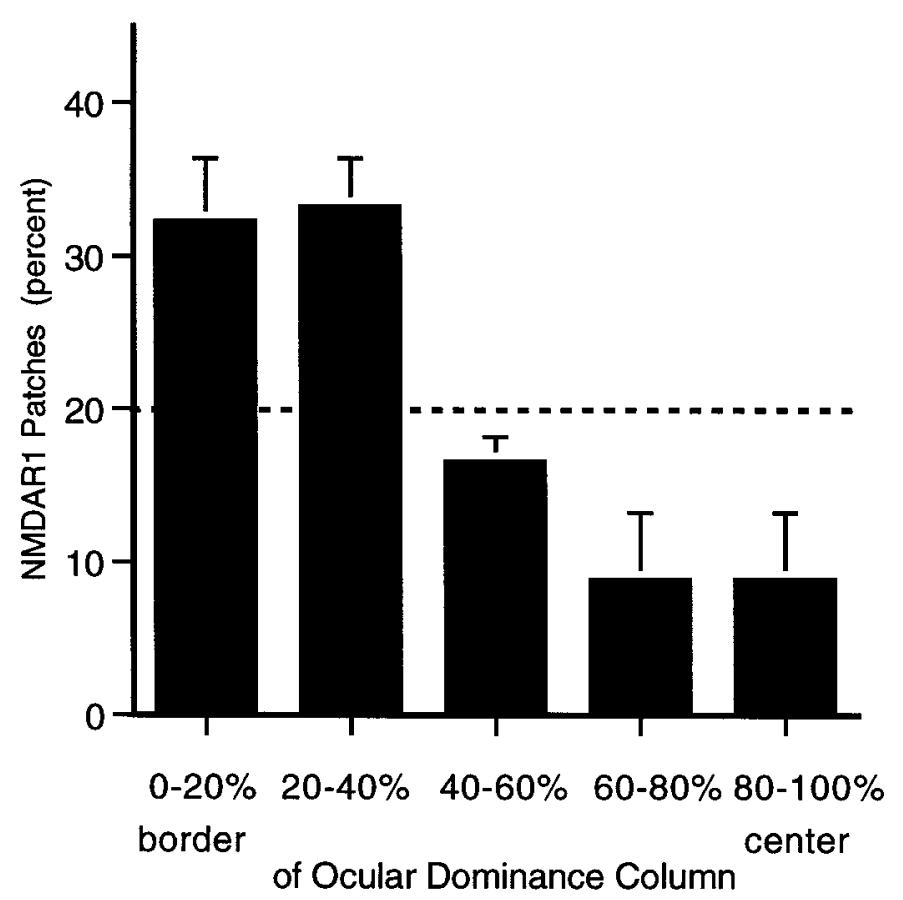

Figure 10. The frequency distribution of NMDAR1 patches located in different regions from the borders $(0-20 \%)$ to the centers $(80-100 \%)$ of the ocular dominance bands. The expected frequency is denoted by the dotted line (20\%). Approximately $65 \%$ of the patches were found near the borders $(0-20$ and $20-40 \%)$, and just $18 \%$ of the patches were located near the centers $(60-80$ and $80-100 \%)$ of the ocular dominance bands $\left(p<0.00001 ; \chi^{2}\right.$ test $)$. Error bars represent SEM.

of binocular vision after monocular deprivation to 5 weeks of age. This is the peak of the critical period when there is substantial plasticity in the visual cortex and significant anatomical changes can occur within just a few days of altered visual experience (Antonini and Stryker, 1993). In this case, the altered visual experience was the initiation of binocular experience by simply opening the deprived eye. Just a few days of binocular vision was sufficient to promote expression of the NMDAR1 patches, thereby suggesting a link between the patches and binocular visual experience. Moreover, the patches had a spacing comparable with the width of one eye's ocular dominance columns and a tendency to be located near the borders of ocular dominance columns. It is important to remember that the relationship between NMDAR1 patches and ocular dominance borders was visualized after monocular deprivation; therefore, it will be necessary to extend this analysis to normally reared animals to address this issue definitively.

\section{Relationship to other patchy markers}

Several features intrinsic to the cat visual cortex are arranged in a patchy manner. Most notable are the cytochrome oxidase blobs (Murphy et al., 1990, 1991, 1995) and the periodic clusters of intracortical connections in the supragranular layers of cat visual cortex (Callaway and Katz, 1990). During development, various other markers including adenosine (Schoen et al., 1990), zinc (Dyck et al., 1993), and serotonin (Dyck and Cynader, 1993) are transiently patchy. The arrangement of these markers is of particular interest because of their association to the NMDA receptor. It is known that there is a distinct binding site for zinc on the NMDA receptor, that nitric oxide and NMDAR1-IR are colocalized on spines (Aoki et al., 1997), and that zinc alone
(Forsythe et al., 1988) and serotonin in conjunction with nitric oxide (Montague et al., 1994; Kara and Friedlander, 1995) have been shown to modulate NMDA receptor-mediated excitatory transmission. Consequently, the distribution of the NMDA receptor may be the common element linking these patchy anatomical markers.

Both the NMDAR1 patches and the cytochrome oxidase blobs are found in the supragranular layers; however, they are related to different aspects of ocular dominance columns. Blobs are spatially linked with the centers of ocular dominance columns (Horton, 1984; Murphy et al., 1995; Hubener et al., 1997) and functionally linked with the columnar organization of the visual cortex and the streaming of information into parallel processing pathways (Zeki, 1976; Livingstone and Hubel, 1983, 1984; DeYoe and Van Essen, 1985, 1988; Shipp and Zeki, 1985; Murphy et al., 1991, 1995; Van Essen et al., 1992; Shoham et al., 1997). Furthermore, blobs develop even in the absence of retinal activity (Kuljis and Rakic, 1990), suggesting that their arrangement is insensitive to experience-dependent change. The NMDAR1 patches, on the other hand, are sensitive to changes in the pattern of retinally driven activity and tend to be located near the borders of ocular dominance columns. Perhaps the blobs and NMDAR1 patches fill complementary niches during the development of visual cortical columns, possibly contributing in a synergistic manner to the patterning of ocular dominance columns.

\section{Role for NMDAR1 patches in column development}

What role might be served by a patchy distribution of the NMDA receptor in the developing visual cortex? It is curious that when the NMDAR1 patches first appear at 2 weeks of age, they are found in the only lamina that never receives direct thalamic input (Shatz and Luskin, 1986), the same area where the clustered intracortical connections emerge (Callaway and Katz, 1990). This suggests that, initially, the NMDAR1 patches are not directly driven by geniculocortical inputs but instead reflect an organization intrinsic to the visual cortex that interacts with retinally driven activity. It has been proposed that the presence of NMDA receptors is important for the formation and strengthening of nascent synapses (Aoki et al., 1994; Aoki, 1997) and that their arrangement may act as a blueprint for developing synapses (Durand et al., 1996). Based on these ideas, the NMDAR1 patches could be an element of an intrinsic columnar protomap. A role for the patches in such a map can be formulated by considering the model for NMDA receptor involvement in activity-dependent refinement of developing connections proposed by Cline and Constantine-Paton (1990). In their model, increased NMDA receptor activation leads to the sculpting of axons by synapse stabilization, survival of axon arbors, and axon restriction. Because activation of the NMDA receptor occurs when there is simultaneous pre- and postsynaptic activity (Bliss and Collingridge, 1993), regions with more NMDA receptors could effectively have both a lower threshold for detecting coactive inputs and a greater probability of initiating synapse stabilization and axon restriction. The regions of binocular overlap in cat V1 are primarily near column borders (Hata and Stryker, 1994), have lower levels of coactivity, and are where connections are sculpted and refined during experience-dependent development of the columns. The NMDAR1 patches were not found when cortical binocularity and normal ocular dominance column development were disrupted by monocular deprivation; however, they were present when binocular vision was introduced. These results suggest a relationship between the NMDAR1 patches and 
the ocular dominance columns, although further experiments will be necessary to answer whether the NMDAR1 patches are a factor in the normal development of ocular dominance columns.

Although normal binocular experience, coupled with activation of the NMDA receptor, is crucial for the development of ocular dominance columns, it is clear that these factors alone cannot account for all of their attributes. For example, Horton and Hocking (1996) have demonstrated an adult-like pattern of ocular dominance columns at birth in the macaque. These results support the notion that factors intrinsic to the cortex, interacting with retinal activity, guide the overall arrangement of cortical columns (Jones et al., 1991). The NMDAR1 patches have the appropriate characteristics to be involved in guiding the $2 \mathrm{D}$ arrangement of nascent columns.

\section{REFERENCES}

Anderson PA, Olavarria J, Van Sluyters RC (1988) The overall pattern of ocular dominance bands in cat visual cortex. J Neurosci 8:2183-2200.

Antonini A, Stryker MP (1993) Rapid remodeling of axonal arbors in the visual cortex. Science 260:1819-1821.

Aoki C (1997) Postnatal changes in the laminar and subcellular distribution of NMDA-R1 subunits in the cat visual cortex as revealed by immuno-electron microscopy. Dev Brain Res 98:41-59.

Aoki C, Venkatesan C, Go C-G, Mong JA, Dawson TM (1994) Cellular and subcellular localization of NMDA-R1 subunit immunoreactivity in the visual cortex of adult and neonatal rats. J Neurosci 14:5202-5222.

Aoki C, Rhee J, Lubin M, Dawson TM (1997) NMDA-R1 subunit of the cerebral cortex co-localizes with neuronal nitric oxide synthase at preand postsynaptic sites and in spines. Brain Res 750:25-40.

Artola A, Singer W (1987) Long-term potentiation and NMDA receptors in rat visual cortex. Nature 330:649-652.

Artola A, Singer W (1990) The involvement of $N$-methyl-D-aspartate receptors in induction and maintenance of long-term potentiation in rat visual cortex. Eur J Neurosci 2:254-269.

Bear MF, Kleinschmidt A, Gu Q, Singer W (1990) Disruption of experience-dependent synaptic modifications in striate cortex by infusion of an NMDA-receptor antagonist. J Neurosci 10:909-925.

Bear MF, Press WA, Conners BW (1992) Long-term potentiation in slices of kitten visual cortex and the effects of NMDA receptor blockade. J Neurophysiol 67:1-11.

Bliss TVP, Collingridge GL (1993) A synaptic model of memory: longterm potentiation in the hippocampus. Nature 361:31-39.

Bode-Greuel KM, Singer W (1989) The development of $N$-methyl-Daspartate receptors in cat visual cortex. Dev Brain Res 46:197-204.

Callaway EM, Katz LC (1990) Emergence and refinement of clustered horizontal connections in cat striate cortex. J Neurosci 10:1134-1153.

Catalano SM, Chang CK, Shatz CJ (1997) Activity-dependent regulation of NMDAR1 immunoreactivity in the developing visual cortex. J Neurosci 17:8376-8390.

Cline HT, Constantine-Paton M (1990) NMDA receptor agonist and antagonists alter retinal ganglion cell arbor structure in the developing frog retinotectal projection. J Neurosci 10:1197-1216.

Collingridge GL, Singer W (1990) Excitatory amino acid receptors and synaptic plasticity. Trends Pharmacol Sci 11:290-296.

Constantine-Paton M, Cline HT, Debski E (1990) Patterned activity, synaptic convergence, and the NMDA receptor in developing visual pathways. Annu Rev Neurosci 13:129-154.

Daw NW (1994) Mechanisms of plasticity in the visual cortex. Invest Ophthalmol Vis Sci 35:4168-4179.

DeYoe EA, Van Essen DC (1985) Segregation of efferent connections and receptive field properties in visual area V2 of the macaque. Nature 317:58-61.

DeYoe EA, Van Essen DC (1988) Concurrent processing streams in monkey visual cortex. Trends Neurosci 5:219-226.

Durand GM, Kovalchuk Y, Konnerth A (1996) Long-term potentiation and functional synapse induction in developing hippocampus. Nature 381:71-75.

Dyck RH, Cynader MS (1993) An interdigitated columnar mosaic of cytochrome oxidase, zinc, and neurotransmitter-related molecules in cat and monkey visual cortex. Proc Natl Acad Sci USA 90:9066-9069.

Dyck RH, Beaulieu C, Cynader MS (1993) Histochemical localization of synaptic zinc in the developing cat visual cortex. J Comp Neurol 329:53-67.

Forsythe ID, Westbrook GL, Mayer ML (1988) Modulation of excitatory synaptic transmission by glycine and zinc in cultures of mouse hippocampal neurons. J Neurosci 8:3733-3741.

Fox K, Daw NW (1993) Do NMDA receptors have a critical function in visual cortical plasticity? Trends Neurosci 16:116-122.

Fox K, Daw N, Sato H, Czepita D (1991) Dark rearing delays the decline in NMDA-receptor efficacy in kitten visual cortex. Nature 350:342-344.

Goelet P, Castellucci VF, Schacher S, Kandel ER (1986) The long and short of long-term memory-a molecular framework. Nature 322: 419-422.

Goodman CS, Shatz CJ (1993) Developmental mechanisms that generate precise patterns of neuronal connectivity. Cell 72:77-98.

Gordon B, Daw N, Parkinson D (1991) The effect of age on binding of MK-801 in the cat visual cortex. Dev Brain Res 62:61-67.

Gordon B, Pardo D, Conant K (1996) Laminar distribution of MK-801, kainate, AMPA, and muscimol sites in cat visual cortex: a developmental study. J Comp Neurol 365:466-478.

Greenamyre JT, Olson JMM, Penney JB, Young AB (1985) Autoradiographic characterization of $N$-methyl-D-aspartate-, quisqualate- and kainate-sensitive glutamate binding sites. J Pharmacol Exp Ther 233:254-263.

Gu Q, Bear MF, Singer W (1989) Blockade of NMDA receptors prevents ocularity changes in kitten striate cortex after reversed monocular deprivation. Dev Brain Res 47:281-288.

Guibas L, Stolfi J (1985) Primitives for the manipulation of general subdivisions and the computation of Voronoi diagrams. ACM Trans Graphics 4:74-123.

Hata Y, Stryker MP (1994) Control of thalamocortical afferent rearrangement by postsynaptic activity in developing visual cortex. Science 265:1732-1735.

Hebb DO (1949) The organization of behavior. New York: Wiley.

Horton JC (1984) Cytochrome oxidase patches: a new cytoarchitectonic feature of monkey visual cortex. Philos Trans R Soc Lond [Biol] 304:199-253.

Horton JC, Hocking DR (1996) An adult-like pattern of ocular dominance columns in striate cortex of newborn monkeys prior to visual experience. J Neurosci 16:1791-1807.

Horton JC, Hubel DH (1981) Regular patchy distribution of cytochrome oxidase blobs in primary visual cortex of macaque monkey. Nature 292:762-764.

Hubel DH, Wiesel TN (1965) Binocular interaction in striate cortex of kittens reared with artificial squint. J Neurophysiol 28:1041-1059.

Hubel DH, Wiesel TN (1968) Receptive fields and functional architecture of monkey striate cortex. J Physiol (Lond) 195:215-243.

Hubel DH, Wiesel TN (1970) The period of susceptibility to the physiological effects of unilateral eye closure in kittens. J Physiol (Lond) 206:419-436.

Hubener M, Shoham D, Grinvald A, Bonhoeffer T (1997) Spatial relationship among three columnar systems in cat area 17. J Neurosci 17:9270-9284.

Huntley GW, Vickers JC, Janssen W, Brose N, Heinemann SF, Morrison JH (1994) Distribution and synaptic localization of immunocytochemically identified NMDA receptor subunit proteins in sensorymotor and visual cortices of monkey and human. J Neurosci 14: 3603-3619.

Jones DG, Van Sluyters RC, Murphy KM (1991) A computational model for the overall pattern of cortical ocular dominance. J Neurosci 11:3794-3808.

Kara P, Friedlander MJ (1995) The role of nitric oxide in modulating the visual response of neurons in the cat striate cortex. Soc Neurosci Abstr 21:1753.

Kato N, Artola A, Singer W (1991) Developmental changes in the susceptibility to long-term potentiation of neurons in rat visual cortex slices. Dev Brain Res 60:43-50.

Kirkwood A, Bear MF (1994) Hebbian synapses in visual cortex. J Neurosci 14:1634-1645.

Kirkwood A, Hey-Kyoung L, Bear MF (1995) Co-regulation of longterm potentiation and experience-dependent synaptic plasticity in visual cortex by age and experience. Nature 375:328-331.

Kirkwood A, Rioult MG, Bear MF (1996) Experience-dependent modification of synaptic plasticity in visual cortex. Nature 381:526-528.

Kleinschmidt A, Bear MF, Singer W (1987) Blockade of "NMDA" 
receptors disrupts experience-dependent plasticity of kitten striate cortex. Science 238:355-358.

Komatsu Y, Toyama K (1989) Long-term potentiation of excitatory synaptic transmission in kitten visual cortex. Biomed Res 10:57-59.

Komatsu Y, Toyama K, Maeda J, Sakaguchi H (1981) Long-term potentiation investigated in a slice preparation of striate cortex of young kittens. Neurosci Lett 26:269-274.

Komatsu Y, Fujii K, Maeda J, Sakaguchi H, Toyama K (1988) Longterm potentiation of synaptic transmission in kitten visual cortex. J Neurophysiol 59:124-141.

Komatsu Y, Nakajima S, Toyama K (1991) Induction of long-term potentiation without the participation of $N$-methyl-D-aspartate receptors in kitten visual cortex. J Neurophysiol 65:20-32.

Kuljis RO, Rakic P (1990) Hypercolumns in primate visual cortex can develop in the absence of cues from photoreceptors. Proc Natl Acad Sci USA 87:5303-5306.

LeVay S, Connolly M, Houde J, Van Essen DC (1985) The complete pattern of ocular dominance stripes in the striate cortex and visual field of the macaque monkey. J Neurosci 5:486-501.

Livingstone MS, Hubel DH (1983) Specificity of cortico-cortical connections in monkey visual system. Nature 304:531-534.

Livingstone MS, Hubel DH (1984) Anatomy and physiology of a color system in the primate visual cortex. J Neurosci 4:309-356.

Montague PR, Gancayco CD, Winn MJ, Marchase RB, Friedlander MJ (1994) Role of NO production in NMDA receptor-mediated neurotransmitter release in cerebral cortex. Science 263:973-977.

Movshon JA, Van Sluyters RC (1981) Visual neural development. Annu Rev Psychol 32:477-522.

Murphy KM, Mitchell DE (1987) Reduced visual acuity in both eyes of monocularly deprived kittens following a long or short period of reverse occlusion. J Neurosci 7:1526-1536.

Murphy KM, Van Sluyters RC, Jones DG (1990) Cytochrome-oxidase in cat visual cortex: is it periodic? Soc Neurosci Abstr 16:292.

Murphy KM, Van Sluyters RC, Jones DG (1991) Cytochrome-oxidase blobs in cat visual cortex. Invest Ophthalmol Vis Sci 32:1116.

Murphy KM, Jones DG, Van Sluyters RC (1995) Cytochrome-oxidase blobs in cat primary visual cortex. J Neurosci 15:4196-4208.

Murphy KM, Trepel C, Pegado VD (1996) Non-uniform distribution of the NMDAR1 receptor subunit in kitten visual cortex at the peak of the critical period. Mol Vis [On-line] 2:9. Available: http://www.emory. edu/MOLECULAR_VISION/v2/murphy.

Olavarria J, Van Sluyters RC (1985) Unfolding and flattening the cortex of gyrencephalic brains. J Neurosci Methods 15:191-202.

Olson CR, Freeman RD (1980) Profile of the sensitive period for monocular deprivation in kittens. Exp Brain Res 39:17-21.

Perkins AT, Teyler TJ (1988) A critical period for long-term potentiation in the developing rat visual cortex. Brain Res 439:222-229.

Reynolds IJ, Bear MF (1991) Effects of age and visual experience on ${ }^{3} \mathrm{H}$ MK801 binding to NMDA receptors in the kitten visual cortex. Exp Brain Res 85:611-615.
Rosier AM, Arckens L, Orban GA, Vandesande F (1993) Laminar distribution of NMDA receptors in cat and monkey visual cortex visualized by [ $\left.{ }^{3} \mathrm{H}\right]-\mathrm{MK}-801$ binding. J Comp Neurol 335:369-380.

Schoen SW, Leutencker B, Kreutzberg GW, Singer W (1990) Ocular dominance plasticity and developmental changes of 5 '-nucleotidase distributions in the kitten visual cortex. J Comp Neurol 296:379-392.

Shatz CJ, Luskin MB (1986) The relationship between the geniculocortical afferents and their cortical target cells during development of the cat's primary visual cortex. J Neurosci 6:3655-3667.

Shipp S, Zeki S (1985) Segregation of pathways leading from area V2 to areas V4 and V5 of macaque monkey visual cortex. Nature 315: 322-325.

Shoham D, Hubener M, Schulze S, Grinvald A, Bonhoeffer, T (1997) Spatio-temporal frequency domains and their relation to cytochrome oxidase staining in cat visual cortex. Nature 385:529-533.

Siegel SJ, Brose N, Janssen WG, Gasic GP, Jahn R, Heinemann SF, Morrison JH (1994) Regional, cellular and ultrastructural distribution of the glutamate receptor subunit NMDAR1 in monkey hippocampus. Proc Natl Acad Sci USA 91:564-568.

Singer W, Artola A (1991) The role of NMDA receptors in usedependent synaptic plasticity of the visual cortex. In: Excitatory amino acids synaptic function (Wheal HV, Thomson AM, eds), pp 333-353. London: Academic.

Smith AL, Thompson ID (1994) Distinct laminar differences in the distribution of excitatory amino acid receptors in adult ferret primary visual cortex. Neuroscience 61:467-479.

Sutor B, Hablitz JJ (1989) Long-term potentiation in frontal cortex: role of NMDA-modulated polysynaptic excitatory pathways. Neurosci Lett 97:111-117.

Teyler T, Aroniadou V, Berry RL, Borroni A, DiScenna P, Grover L, Lambert N (1990) LTP in neocortex. Semin Neurosci 2:365-379.

Trepel C, Pegado VD, Murphy KM (1996) Patchy distribution of the NMDAR1 receptor subunit in developing kitten visual cortex. Invest Ophthalmol Vis Sci 37:S480.

Tsumoto T, Suda K (1979) Cross-depression: an electrophysiological manifestation of binocular competition in the developing visual cortex. Brain Res 168:190-194.

Upton GJG, Fingleton B (1985) Spatial data analysis by example: point pattern and quantitative data. New York: Wiley.

Van Essen DC, Andersen CH, Felleman DJ (1992) Information processing in the primate visual system: an integrated systems perspective. Science 255:419-423.

Wong-Riley M (1979) Changes in the visual system of monocularly sutured or enucleated cats demonstrable with cytochrome oxidase histochemistry. Brain Res 171:11-28.

Zeki SM (1976) The functional organization of projections from striate to prestriate visual cortex in the rhesus monkey. Cold Spring Harb Symp Quant Biol 40:591-600. 